\title{
A GENERALIZATION OF THE VIETORIS-BEGLE THEOREM
}

\author{
JERZY DYDAK AND GEORGE KOZLOWSKI
}

(Communicated by Doug W. Curtis)

\begin{abstract}
A theorem is proved which generalizes both the Vietoris-Begle theorem and the cell-like theorem for spaces of finite defomation dimension. The proof is geometric and uses a double mapping cylinder trick.
\end{abstract}

The double mapping cylinder $\operatorname{DM}(f)$ of a map $f: X^{\prime} \rightarrow X$ is the space

$$
X^{\prime} \times[-1,1] \cup_{a} X \times\{-1,1\},
$$

where $a(x, i)=(f(x), i)$ for $i=-1,1$. The composition $p(f \times$ id $): X^{\prime} \times[-1,1] \rightarrow X$ (here $p: X \times[-1,1] \rightarrow X$ is the projection) induces $\hat{f}: \operatorname{DM}(f) \rightarrow X$ such that $\hat{f}^{-1}(x)$ is the suspension $\sum f^{-1}(x)$ of $f^{-1}(x)$ for all $x \in X$.

If $f: X^{\prime} \rightarrow X$ and $A \subset X$, then $f \mid f^{-1}(A): f^{-1}(A) \rightarrow A$ is denoted by $f_{A}$.

We are going to use a double mapping cylinder trick as in $\left[\mathbf{K}_{\mathbf{1}}\right.$, Lemma 7$]$ or $\left[\mathbf{K}_{\mathbf{2}}\right.$, Lemma 2] (see also [ $\mathbf{K}_{\mathbf{3}}, \mathbf{D}-\mathbf{S}_{\mathbf{1}}$, and $\mathbf{D}-\mathbf{S}_{\mathbf{2}}$, p. 139]):

If $\hat{f}^{\#}:[X, E] \rightarrow[\mathrm{DM}(f), E]$ is a surjection then $f^{\#}:[X, E] \rightarrow\left[X^{\prime}, E\right]$ is an injection.

Indeed, let $g, h: X \rightarrow E$ be two maps such that $g f \approx h f$. Then there is a map $H: \operatorname{DM}(f) \rightarrow E$ such that $H(x,-1)=g(x)$ and $H(x, 1)=h(x)$ for $x \in X$. Since $\hat{f}^{\#}$ is surjective, $H$ extends over the mapping cylinder $M(\hat{F})$ of $\hat{f}$. Let $F: M(\hat{f}) \rightarrow E$ be an extension of $H$. Notice that $\hat{f} \mid X \times\{i\}$ is a homeomorphism for $i=-1,1$. Using this one can produce an embedding $a: X \times[-1,1] \rightarrow M(\hat{f})$ such that $a \mid X \times\{i\}=\mathrm{id}$ for $i=-1,1$. Then $F \cdot a$ is a homotopy joining $g$ and $h$.

The aim of this note is to prove the following

THEOREM. Let $E$ be a connected $C W$ complex and let $f: X^{\prime} \rightarrow X$ be a closed surjective map of paracompact Hausdorff spaces. If all maps $f^{-1}(x) \rightarrow \Omega E$ are nullhomotopic for all $x \in X$ and $\hat{f}_{A}^{\#}:[A, \Omega E] \rightarrow\left[\mathrm{DM}\left(f_{A}\right), \Omega E\right]$ is a surjection for all closed subsets $A$ of $X$, then $\hat{f}_{A}^{\#}:[A, E] \rightarrow\left[\mathrm{DM}\left(f_{A}\right), E\right]$ is a surjection for all closed subsets $A$ of $X$.

If $\hat{f}_{A}^{\#}:[A, E] \rightarrow\left[\mathrm{DM}\left(f_{A}\right), E\right]$ is a surjection for all closed subsets $A$ of $X$, then the ir.zage of $f^{\#}:[X, E] \rightarrow\left[X^{\prime}, E\right]$ is the set

$$
\left\{[g]: g: X^{\prime} \rightarrow E \text { and } g \mid f^{-1}(x) \approx \text { const for all } x \in S\right\} .
$$

Before proceeding with the proof of the Theorem let us draw certain corollaries to it.

Received by the editors October 21, 1986.

1980 Mathematics Subject Classification (1985 Revision). Primary 55N05; Secondary 55N20, 55 P20.

Key words and phrases. Vietoris-Begle theorem, double mapping cylinder, loop space. 
COROLlaRY 1. Let $E$ be a connected $C W$ complex such that $\pi_{i} E=0$ for $i>p$. If $f: X^{\prime} \rightarrow X$ is a closed surjective map of paracompact Hausdorff spaces such that all maps $f^{-1}(x) \rightarrow \Omega^{k} E$ are nullhomotopic for all $x \in X$ and for $1 \leq k \leq p$, then $f^{\#}:[X, E] \rightarrow\left[X^{\prime}, E\right]$ is injective and its image is the set

$$
\left\{[g]: g: X^{\prime} \rightarrow E \text { and } g \mid f^{-1}(x) \approx \text { const for all } x \in X\right\} \text {. }
$$

ProOF. $\pi_{i}\left(\Omega^{p} E\right)=\pi_{i+p} E=0$ for $i>0$. Therefore each component of $\Omega^{p} E$ is contractible, and since point inverses of $\hat{f}_{A}$ are connected, $\hat{f}_{A}^{\#}:\left[A, \Omega^{p} E\right] \rightarrow$ $\left[\mathrm{DM}\left(f_{A}\right), \Omega^{p} E\right]$ is a surjection for each closed subset $A$ of $X$. By induction we get from the Theorem that $\hat{f}_{A}^{\#}:\left[A, \Omega^{k} E\right] \rightarrow\left[\mathrm{DM}\left(f_{A}\right), \Omega^{k} E\right]$ is a surjection for $0 \leq k \leq p$ and the double mapping cylinder trick implies Corollary 1 .

REMARK. Corollary 1 strengthens and gives a simpler proof of one of the basic theorems used in the proof of Theorem 2 of $\left[\mathbf{K}_{\mathbf{4}}\right]$. A statement and proof by John Walsh for compact metric spaces along with some further discussion can be found in Appendix B of [W].

If one interprets the reduced Čech cohomology group $\tilde{H}^{q}(A ; G)$ as $[A, K(G, q)]$ (see $[\mathbf{G}$ or $\mathbf{S}]$ ), then Corollary 1 implies the Vietoris-Begle theorem which we recall in the improved version from [D] (see also [S, p. 344]):

VIETORIS-BEGLE THEOREM. Suppose $f: X^{\prime} \rightarrow X$ is a closed surjective map of paracompact Hausdorff spaces such that $\tilde{H}^{q}\left(f^{-1}(x) ; G\right)=0$ for all $x \in X$ and for $q<n(n \geq 0)$. Then the sequence

$$
0 \rightarrow H^{q}(X ; G) \stackrel{H^{q}(f)}{\longrightarrow} H^{q}\left(X^{\prime} ; G\right) \stackrel{\gamma}{\rightarrow} \prod_{x \in X} H^{q}\left(f^{-1}(x) ; G\right)
$$

is exact for $q \leq n$, where $\gamma$ is induced by the inclusion induced homomorphisms $\tilde{H}^{q}\left(X^{\prime} ; G\right) \rightarrow \tilde{H}^{q}\left(f^{-1}(x) ; G\right)$.

REMARK. The assertion that $\hat{f}^{\#}:[X, E] \rightarrow[\mathrm{DM}(f), E]$ is a surjection means that if $g, h$ are maps from $X$ into $E$ and if $H: X^{\prime} \times I \rightarrow E$ is a homotopy between $g f$ and $h f$, then $H$ is homotopic rel. $X^{\prime} \times\{0,1\}$ to a homotopy $K: X^{\prime} \times I \rightarrow E$ which induces a homotopy $X \times I \rightarrow E$ between $g$ and $h$. The statement of the Vietoris-Begle theorem can therefore be strengthened to include a corresponding assertion, as can be seen in the proof of that theorem.

COROLlARY 2. If $f: X^{\prime} \rightarrow X$ is a closed surjective map between paracompacta of finite defomation dimension such that $f^{-1}(x)$ has trivial shape for each $x \in X$, then $f$ is a shape equivalence.

Proof. We must show that for any CW complex $E, f^{\#}:[X, E] \rightarrow\left[X^{\prime}, E\right]$ is a bijection. By Corollary 1 it is so for all $E$ such that almost all its homotopy groups are trivial. Let $n>\operatorname{def}-\operatorname{dim} X$, def-dim $X^{\prime}$ (see $\left[\mathbf{D}-\mathbf{S}_{\mathbf{2}}\right]$ for a definition of the deformation dimension). By attaching $k$-cells for $k>n$ to $E$ we can construct $E^{\prime}$ such that $\pi_{i} E^{\prime}=0$ for $i>n$. Since the $n$-skeletons of $E$ and of $E^{\prime}$ coincide, the inclusion $i: E \rightarrow E^{\prime}$ induces bijections $[X, E] \rightarrow\left[X, E^{\prime}\right]$ and $\left[X^{\prime}, E\right] \rightarrow\left[X^{\prime}, E^{\prime}\right]$. Now, $[X, E] \rightarrow\left[X^{\prime}, E\right]$ is a bijection because $\left[X, E^{\prime}\right] \rightarrow\left[X^{\prime}, E^{\prime}\right]$ is a bijection.

REMARK. See $\left[K_{1,3}, \mathbf{S h}\right.$, and D-S $]$ for other proofs of results similar to Corollary 2.

In the process of proving the Theorem we need to replace $E$ by a homotopically equivalent space which is an ANE for paracompact spaces. 
LEMma. Any CW complex has the homotopy type of an ANE for paracompact spaces.

ProOF. Take a simplicial complex $K$ such that its geometric realization $|K|$ with the metric topology is homotopy equivalent to a given $\mathrm{CW}$ complex (see [G, p. 149]). $|K|$ is considered as a subset of $l_{2}\left(K^{(0)}\right)$ with the metric induced from $l^{2}\left(K^{(0)}\right)$. As in $\left[\mathbf{H}\right.$, p. 107] the space $\left|K^{(n)}\right|$ is complete for each $n$. Therefore the subset

$$
X=\bigcup_{n=0}^{\infty}\left|K^{(n)}\right| \times[n, \infty)
$$

of $|K| \times[0, \infty)$ is complete in the metric induced from the obvious metric on $l_{2}\left(K^{(0)}\right) \times[0, \infty)$. It is clear that the inclusion $X \rightarrow|K| \times[0, \infty)$ induces isomorphisms of homotopy groups and, by the Whitehead theorem, it is a homotopy equivalence. Since every complete metric ANR is an ANE for paracompact spaces (see [H, pp. 84, 87]), the Lemma follows.

PROOF OF THEOREM. Assume $E$ is an ANE for paracompact spaces. The two statements we have to prove will have the same proof in the beginning. So let us assume that $F: Y \rightarrow X$ is a closed surjective map, where $Y$ is paracompact Hausdorff, and suppose $g: Y \rightarrow E$ is a map such that $g \mid F^{-1}(x)$ is nullhomotopic for all $x$ in $X$. Let $p: M(F) \rightarrow X$ be the projection.

Fix $x \in X$. Since $g \mid F^{-1}(x) \approx$ const, there exists an extension $g^{\prime}: Y \cup p^{-1}(x) \rightarrow E$ of $g$. Define $g^{\prime \prime}: Y \cup p^{-1}(x) \cup X \rightarrow E$ by $g^{\prime \prime} \mid Y \cup p^{-1}(x)=g^{\prime}$ and $g^{\prime \prime}(X)=g^{\prime}(x)$. $g^{\prime \prime}$ extends over a neighborhood $U$ of $Y \cup p^{-1}(x) \cup X$ in $M(F)$. Choose a neighborhood $V_{x}$ of $x$ in $X$ such that $p^{-1}\left(V_{x}\right) \subset U$. Having done that for all $x$ in $X$, we choose a locally finite cover $\left\{A_{s}\right\}_{s \in S}$ consisting of closed sets which is a refinement of $\left\{V_{x}\right\}_{x \in X}$. Then, for each $s \in S$, we choose a map $g_{s}: Y \cup p^{-1}\left(A_{s}\right) \rightarrow E$ such that $g_{s} \mid Y=g$ and $g_{s}\left(A_{s}\right)$ is a one-point set.

Claim. Suppose $F=f$ or $F=\hat{f}$. If $A$ is a closed subset of $A_{s}$ for some $s$ in $S$ and $h: p^{-1}(A) \rightarrow E$ is an extension of $g \mid F^{-1}(A)$, then

$$
h \approx g_{s} / p^{-1}(A) \text { rel. } F^{-1}(A) .
$$

The Claim will be proved by showing that the map

$$
c: B=F^{-1}(A) \times[-1,1] \cup p^{-1}(A) \times\{-1,1\} \rightarrow E
$$

defined by $c(x, t)=g(x)$ for $(x, t) \in F^{-1}(A) \times[-1,1], c(X,-1)=g_{s}(x), c(x, 1)=$ $h(x)$ for $x \in p^{-1}(A)$, is nullhomotopic (because $c$ will be extendible over $p^{-1}(A) \times$ $[-1,1]$ in this case, which will give a homotopy from $g_{s}$ to $h$ over $\left.F^{-1}(A)\right)$.

Assuming that the Claim holds we proceed as follows:

If $a: Y \cup p^{-1}(D) \rightarrow E$ is an extension of $g(D$ is closed in $X)$ and $s \in S$, then $a\left|p^{-1}\left(D \cap A_{s}\right) \approx g_{s}\right| p^{-1}\left(D \cap A_{s}\right)$ rel. $F^{-1}\left(D \cap A_{s}\right)$. Since $g_{s} \mid F^{-1}\left(A_{s}\right) \cup p^{-1}\left(D \cap A_{s}\right)$ is extendible over $p^{-1}\left(A_{s}\right)$, so is $a \mid F^{-1}\left(A_{s}\right) \cup p^{-1}\left(D \cap A_{s}\right)$. Therefore there exists an extension $a^{\prime}: Y \cup p^{-1}\left(D \cup A_{s}\right) \rightarrow E$ of $a$. By well-ordering $S$ and by the transfinite induction we can construct an extension $g^{\prime}: p^{-1}(X) \rightarrow E$ of $g$.

So it remains to prove the Claim by showing that $c \approx$ const.

Case 1. $F=\hat{f}$.

Here $g_{s} \mid A=$ const impleis $h \mid A \approx$ const by the double mapping cylinder trick. Without loss of generality we may assume $h(A)=g_{s}(A)$. Now $c=c^{\prime} d$, where 
$d: B \rightarrow \sum \operatorname{DM}\left(f_{A}\right)$ is obtained by contracting each of $A \times\{-1\}$ and $A \times\{1\}$ to a point. Let $c^{\prime \prime}: \operatorname{DM}\left(f_{A}\right) \rightarrow \Omega E$ be the map induced by $c^{\prime}$. Since

$$
\hat{f}_{A}^{\#}:[A, \Omega E] \rightarrow\left[\mathrm{DM}\left(f_{A}\right), \Omega E\right]
$$

is a surjection, there is a map $d^{\prime}: A \rightarrow \Omega E$ such that $d^{\prime} \hat{f}_{A} \approx c^{\prime \prime}$. This implies that there is a map $c^{\prime \prime \prime}: \sum A \rightarrow E$ such that $c^{\prime \prime \prime}\left(\sum \hat{f}_{A}\right) \approx c^{\prime}$, and therefore $c \approx$ $c^{\prime \prime \prime}\left(\sum \hat{f}_{A}\right) d$. However $\left(\sum \hat{f}_{A}\right) d=b w$, where $w: B \rightarrow A \times[-1,1]$ is induced by $\hat{f}_{A} \times$ id: $\operatorname{DM}\left(f_{A}\right) \times[-1,1] \rightarrow A \times[-1,1]$ (notice that $B$ is homeomorphic to $\operatorname{DM}\left(\hat{f}_{A}\right)$ ) and $b: A \times[-1,1] \rightarrow \sum A$ is the quotient map. Since $b \approx$ const, we have $\left(\sum \hat{f}_{A}\right) d \approx$ const and, consequently $c \approx$ const (see the diagram). This concludes the proof of the Claim for $F=\hat{f}$. Therefore

$$
\hat{f}_{A}^{\#}:[A, E] \rightarrow\left[\mathrm{DM}\left(f_{A}\right), E\right]
$$

is a surjection.

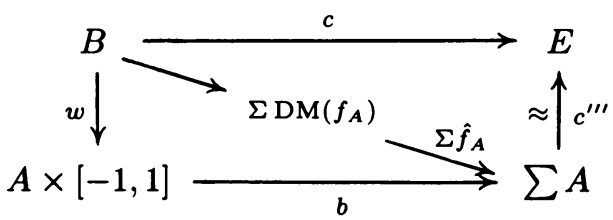

Case 2. $F=f$.

Observe that $B$ is homeomoprhic to $\operatorname{DM}\left(f_{A}\right)$. Therefore $c$ extends over the mapping cylinder of $\hat{f}_{A}$. Since $c \mid A \times\{-1\}=$ const, any such extension is nullhomotopic. Therefore $c \approx$ const.

This completes the proof of the Theorem.

We thank John Walsh for a remark which helped us to find the right formulation of the Theorem.

\section{REFERENCES}

[D] J. Dydak, An addendum to the Vietoris-Begle theorem, Topology Appl. 23 (1986), 75-86.

[D-S $]$ J. Dydak and J. Segal, Strong shape theory, Dissertationes Math. 192 (1981), 1-42.

[D-S $\mathbf{S}_{2}$ ] Shape theory: An introduction, Lecture Notes in Math., vol. 688, Springer-Verlag, Berlin and New York, 1978 pp. 1-150.

[G] B. I. Gray, Homotopy theory, Academic Press, New York 1975.

[H] S. T. Hu, theory of retracts, Wayne State Univ. Press, Detroit, 1965.

[K. $\mathbf{K}_{1}$ G. Kozlowski, Mapping theorems for homotopy, Dissertation, University of Michigan, 1968 (University Microfilms, Ann Arbor, 1968).

$\left[\mathbf{K}_{2}\right]-$, Maps of ANR's determined on null sequences of AR's, Studies in Topology (Proc. Conf., Charlotte, N.C., March 1974). Academic Press, New York 1975, pp. 277-284.

$\left[\mathbf{K}_{\mathbf{3}}\right] \longrightarrow$, Images of ANR's, Trans. Amer. Math. Soc. (to appear).

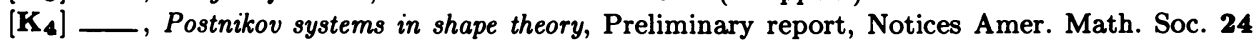
(1977).

[S] E. Spanier Algebraic topology, McGraw-Hill, New York, 1966.

[Sh] R. B. Sher, Realizing cell-like maps in Euclidean spaces, General Topology Appl. 2 (1972), 75-89.

[W] J. J. Walsh, Dimension, cohomological dimension, and cell-like mappings, Shape Theory and Geometric Topology, Lecture Notes in Math., vol. 870, Springer-Verlag, Berlin and New York, 1981, pp. 105-118.

Department of MAThematics, University of TenNessee, KNoxVille, TenesSEE 37996

Department of Mathematics, Auburn University, Auburn, Alabama 36849 